NOTA DE PESQUISA

\title{
CARRAPATOS (ACARI: IXODIDAE) CAPTURADOS NA RESERVA NATURAL DA VALE DO RIO DOCE, LINHARES, ESPÍRITO SANTO*
}

\author{
MARIA OGRZEWALSKA ${ }^{1}$, ALEXANDRE UEZU ${ }^{2}$, FERNANDO FERREIRA ${ }^{1}$, MARCELO B. LABRUNA ${ }^{1}$
}

\begin{abstract}
OGRZEWALSKA, M.; UEZU, A.; FERREIRA, F.; LABRUNA, M.B. [Ticks (Acari: Ixodidae) collected in the Natural Reserve of the Vale do Rio Doce, Linhares, State of Espírito Santo.] Carrapatos (Acari: Ixodidae) capturados na Reserva Natural da Vale do Rio Doce, Linhares, Espírito Santo. Revista Brasileira de Parasitologia Veterinária, v. 16, n. 3, p. 177-179, 2007. Departamento de Medicina Veterinária Preventiva e Saúde Animal, Faculdade de Medicina Veterinária e Zootecnia, Universidade de São Paulo, Av. Prof. Dr. Orlando Marques de Paiva, 87, Cidade Universitária, São Paulo, SP 05508- 270, Brasil. E-mail: labruna@usp.br

We confirm, after decades without new registers, the occurrence of the ticks Amblyomma obolongoguttatum Koch, 1844, A. brasiliense Aragão, 1908, and A. humerale Koch, 1844 in the State of Espírito Santo, Brazil, besides the first record of $A$. naponense (Packard, 1869) in this state.
\end{abstract}

KEY WORDS: Amblyomma, tick, Ixodidae, Brazil.

\section{RESUMO}

O presente trabalho confirma, após décadas sem novos relatos, a ocorrência dos carrapatos Amblyomma obolongoguttatum Koch, 1844, A. brasiliense Aragão, 1908, e A. humerale Koch, 1844 no Estado do Espírito Santo, Brasil, além do primeiro relato de $A$. naponense (Packard,1869) para este estado.

PALAVRAS-CHAVE: Amblyomma, carrapato, Ixodidae, Brasil.

Das 57 espécies de carrapato do gênero Amblyomma que ocorrem na região Neotropical, há 33 no Brasil, parasitando vários animais em diferentes habitats (GUIMARÃES et al., 2001; BARROS-BATTESTI et al., 2006). A Reserva Natural da Vale do Rio Doce (RNVRD) apresenta uma área de cerca de 22 mil hectares ao norte do Estado do Espírito Santo, localizada entre os municípios de Linhares e Jaguaré (1906'-19¹8' S e $39^{\circ} 45^{\prime}-40^{\circ} 19^{\prime}$ W). A vegetação da RNVRD é classificada

*Financiado pela Fapesp (05/56708-5)

${ }^{1}$ Departamento de Medicina Veterinária Preventiva e Saúde Animal, Faculdade de Medicina Veterinária e Zootecnia, Universidade de São Paulo, São Paulo, SP 05508-270. E-mail: labruna@usp.br

${ }^{2}$ Instituto de Pesquisas Ecológicas, Nazaré Paulista, SP como Floresta Estacional Semidecidual de Terras Baixas (IBGE, 1992), conhecida como Floresta de Tabuleiro. O entorno da reserva é muito fragmentado, onde predominam pastagens e cultivos de café, eucalipto e mamão (JESUS; ROLIM, 2005). Neste trabalho, registramos algumas espécies de carrapatos relativamente raras da fauna brasileira, na RNVRD no Estado do Espírito.

Entre 4 e 11 de abril 2006 foram feitas coletas de plantas na RNVRD. Aproveitando esse trabalho, foram coletados também carrapatos encontrados fixados à pele e caminhando sobre as roupas das pessoas, que trabalhavam na mata. Adicionalmente, foram encontrados nas estradas dentro da RNVRD três jabutis, Geochelone denticulata (Linnaeus). Esses foram examinados quanto à presença de carrapatos. Todos os ectoparasitos coletados foram guardados vivos e trazidos para o laboratório do Departamento de Medicina Veterinária Preventiva e Saúde Animal, Universidade de São Paulo, para identificação. Os estádios imaturos foram alimentados em coelhos para a obtenção dos estádios adultos e, em seguida, identificados através de chaves taxonômicas (BARROSBATTESTI et al., 2006). Todos os carrapatos foram depositados na Coleção Nacional dos Carrapatos, Universidade de São Paulo CNC-FMVZ/USP (números do acesso 967 e 968).

Das roupas das pessoas, foram coletados três carrapatos adultos identificados como Amblyomma obolongoguttatum (dois machos e uma fêmea). Das roupas e da pele, foram 
coletadas 12 ninfas de Amblyomma spp, das quais 10 foram criadas até o estádio adulto em laboratório, sendo identificadas como A. obolongoguttatum (quatro fêmeas), A. brasiliense (três fêmeas e dois machos) e Amblyomma naponense (um macho). Não foi possível distinguir individualmente quais ninfas estavam parasitando as pessoas ou quais estavam somente caminhando sobre as roupas. Dos três jabutis examinados, dois estavam infestados por 43 indivíduos adultos de Amblyomma humerale (35 machos e oito fêmeas).

Os registros para A. obolongoguttatum incluem México, Belize, Guatemala, Nicarágua, Costa Rica, Panamá, Colômbia, Suriname, Guiana Francesa, Guiana Inglesa, Venezuela, Peru, Bolívia e os estados brasileiros do Acre, Rondônia, Pará, Mato Grosso, Minas Gerais, Rio de Janeiro e Espírito Santo (GUGLIELMONE et al., 2003; MENDONZA-URIBE; CHÁVEZ-CHOROCCO, 2003; LABRUNA et al., 2005). No Espírito Santo, o último relato datava de mais de 70 anos atrás (ARAGÃO, 1936). Várias espécies de animais silvestres, tais como anta (Tapirus terrestris Linnaeus), porco do mato (Tayassu spp.), carnívoros e animais domésticos, tais como cães, suínos e bovinos, foram relatadas como hospedeiros de A. oblongoguttatum (ROBBINS et al., 1998; LABRUNA et al., 2000; GUIMARÃES et al., 2001; LABRUNA et al., 2002b; LABRUNA et al., 2005; TERRASSINI et al., 2006).

Além de Argentina e Paraguai (GUGLIELMONE et al., 2003), A. brasiliense tem registros de ocorrência para os estados do Rio de Janeiro, São Paulo, Minas Gerais e Pará no Brasil, sendo freqüentemente encontrado nos mamíferos das ordens Artiodactyla e Perissodactyla (ARAGÃO, 1936; GUIMARÃES et al., 2001). O último registro de A. brasiliense no Espírito Santo também foi há mais de 70 anos atrás (ARAGÃO, 1936).

Em relação a A. naponense, seus registros de ocorrência incluem Costa Rica, Panamá, Colômbia, Guiana Francesa, Guiana, Suriname, Venezuela, Equador, Peru, Bolívia e Brasil (GUGLIELMONE et al., 2003). No Brasil, foi encontrado nos estados do Amazonas, Pará, Rondônia, Mato Grosso, Mato Grosso do Sul, Goiás, Minas Gerais, Rio de Janeiro, São Paulo e Paraná (GUIMARÃES et al., 2001; LABRUNA et al., 2005), sendo este o primeiro registro para o estado do Espírito Santo. Essa espécie de carrapato parasita vários mamíferos silvestres de grande e médio porte, no entanto, é mais freqüente em porcos do mato Tayassu spp. Há registros de A. naponense no porco doméstico (ROBBINS et al., 1998; LABRUNA et al., 2002b). Há vários registros de adultos e ninfas de A. obolongoguttatum, A. brasiliense e A. naponense em humanos (JONES et al., 1972; LABRUNA et al., 2000; 2005; GUGLIELMONE et al., 2006; SZABÓ et al., 2006).

Na América do Sul, onde a espécie A. humerale é endêmica, esse carrapato não possui registros válidos apenas para a Argentina, Uruguai, Paraguai e Chile (LABRUNA et al., 2002a; GUGLIELMONE et al., 2003). No Brasil, esta espécie está distribuída pelos estados do Amapá, Amazonas, Acre, Rondônia, Pará, Roraima, Tocantins, Bahia, Minas Gerais e Espírito Santo. Essas são também áreas de ocorrência dos jabotis, G. denticulata e G. carbonaria (Spix), principais hospedeiros dos estádios adultos dessa espécie do carrapato (LABRUNA et al., 2002a). Os estádios imaturos de A. humerale parecem preferir pequenos répteis e mamíferos, tendo LABRUNA et al. 2002a encontrado ninfas em lagartos Plica plica (Linnaeus), Plica umbra (Linnaeus), Kentropyx calcarata Spix e em mamíferos Didelphis marsupialis Linnaeus e Ciclopes didactylus Linnaeus. Nos jabutis capturados, os machos foram encontrados fixados na carapaça, em um aglomerado, sendo que a maioria das fêmeas estava fixada nas pernas, como observado previamente por Labruna et al. (2002a).

O presente trabalho relata o primeiro registro de $A$. naponense no Estado do Espírito Santo e, após décadas sem novos relatos, confirma a presença de $A$. oblongoguttatum, $A$. brasiliense, e A. humerale no estado.

\section{REFERÊNCIAS BIBLIOGRÁFICAS}

ARAGÃO, H. Ixodidas brasileiros e de alguns paizes limitrophes. Memórias do Instituto Oswaldo Cruz, v.31, p.759-843, 1936.

BARROS-BATTESTI, D.M.; ARZUA, M.; BECHARA, G.H. Carrapatos de importância médico-veterinária da Região Neotropical: Um guia ilustrado para identificação de espécies. São Paulo: Vox/ International Consortium on Ticks and Tick-borne Diseases (ICTTD-3)/Butantan, 2006. 223 p.

GUGLIELMONE, A.A.; ESTRADA-PEÑA, A.; KEIRANS, J.E.; ROBBINS, R.G. Ticks (Acari: Ixodida) of the Neotropical Zoogeographic Region. Atalanta, Houten, The Netherlands, International Consortium on Ticks and Tickborne Diseases (ICTTD-2), 2003. 173 p.

GUGLIELMONE, A.A.; BEATI, L.; BARROS-BATTESTI, D.M.; LABRUNA, M.B.; NAVA, S.; VENZAL, J.M.; MANGOLD, A.J.; SZABO, M.P.; MARTINS, J.R.; GONZALEZ-ACUNA, D.; ESTRADA-PENA, A. Ticks (Ixodidae) on humans in South America. Experimental and Applied Acarology, v.40, n. 2, p.83-100, 2006.

GUIMARÃES, J.H.; TUCCI, E.C.; BARROS-BATTESTI, D.M. (Ed.) Ectoparasitos de importância veterinária. São Paulo: Editora Plêidade, Ltda.M.E., 2001. 213 p.

IBGE - FUNDAÇÃO INSTITUTO BRASILEIRO DE GEOGRAFIA E ESTATISTICA. Manual técnico da vegetação brasileira. Rio de Janeiro: IBGE, 1992. 92 p.

JESUS, R.M.; ROLIM, S.G. Fitossociologia da Mata Atlântica de Tabuleiro. Boletim Técnico. SIF, Viçosa, v. 19, p. 1149, 2005.

JONES, E.K.; CLIFFORD, C.M.; KEIRANS, J.E. The ticks of Venezuela (Acarina: Ixodoidea) with a key to the species of Amblyomma in the Western Hemisphere. Brigham Young University Science Bulletin. Biological Series, v. 17, n. 5, p.1-40, 1972.

LABRUNA, M.B.; HOMEM, V.S.F.; HEINEMANN, M.B.; FERREIRA NETO, J.S. Ticks (Acari: Ixodidae) associated 
with rural dogs in Uruará, Eastern Amazon, Brazil. Journal of Medical Entomology, v. 7, n. 5, p. 774-776, 2000.

LABRUNA, M.B.; CAMARGO, L.M.A.; TERRASSINI, F.A.; SCHUMAKER, T.T.S.; CAMARGO, E.P. Notes on Parasitism by Amblyomma humerale (Acari: Ixodidae) in the State of Rondônia, Western Amazon, Brazil. Journal of Medical Entomology, v. 9, n. 6, p. 814-817, 2002a.

LABRUNA, M.B.; CAMARGO, L.M.A.; SCHUMAKER, T.T.S.; CAMARGO, E.P. Parasitism of Domestic Swine (Sus scrofa) by Amblyomma Ticks (Acari: Ixodidae) on a Farm at Monte Negro, Western Amazon. Journal of Medical Entomology, v. 39, n. 1, p. 241-243, 2002 b.

LABRUNA, M.B.; CAMARGO, L.M.A.; TERRASSINI, F.A.; FERREIRA, F.; SCHUMAKER, T.T.S.; CAMARGO, E.P. Ticks (Acari: Ixodidae) from the State of Rondônia, western Amazon, Brazil. Systematic and Applied Acarology, v. 10, n. 1, p. 17-32, 2005.

MENDOZA-URIBE, L.; CHÁVEZ-CHOROCCO, J. Ampliación geográfica de siete especies de Amblyomma
(Acari: Ixodidae) y primer reporte de A. oblongoguttatum Koch, 1844 para Perú. Revista Peruana de Entomologia, v. 44, p. 69-72, 2004.

ROBBINS, R.G.; KARESH, W.B.; PAINTER, R.L.E.; ROSENBERG, S. Ticks of the genus Amblyomma (Acari: Ixododa: Ixodidae) from white-lipped peccaries, Tayassu pecari, in northeastern Bolivia, with comments on host specificity. Entomological News, v. 109, n. 3, p. 172-176, 1998.

SZABÓ, M.P.J.; LABRUNA, M.B.; CASTAGNOLLI, K.C.; GARCIA, M.V.; PINTER, A.; VERONEZ, V.A.; MAGALHÃES, G.M.; CASTRO, M.B.; VOGLIOTTI, A. Ticks (Acari: Ixodidae) parasitizing humans in an Atlantic rainforest reserve of Southeastern Brazil: notes on host suitability. Experimental and Applied Acarology, v.39, n. 3-4, p. 338-346, 2006.

TERRASSINI, F.A.; CAMARGO, L.M.A.; LABRUNA, M.B. Diversidade, taxonomia e manejo de ácaros neotropicais. In: SIMPÓSIO BRASILEIRO DE ACAROLOGIA, 1, 2006, Viçosa. Anais...Viçosa, 2006. p. 144.

Recebido em 15 de fevereiro de 2007.

Aceito para publicação em 30 de julho de 2007. 